\title{
Molecular contrast optical coherence tomography: SNR comparison of techniques and introduction of ground state recovery pump-probe OCT
}

Brian E. Applegate, Marinko V. Sarunic, Changhuei Yang, Joseph A. Izatt

Brian E. Applegate, Marinko V. Sarunic, Changhuei Yang, Joseph A. Izatt, "Molecular contrast optical coherence tomography: SNR comparison of techniques and introduction of ground state recovery pump-probe OCT," Proc. SPIE 5690, Coherence Domain Optical Methods and Optical Coherence Tomography in Biomedicine IX, (13 April 2005); doi: 10.1117/12.592866

SPIE. Event: SPIE BiOS, 2005, San Jose, CA, United States 


\title{
Molecular Contrast Optical Coherence Tomography: SNR Comparison of Techniques and Introduction of Ground State Recovery Pump-Probe OCT
}

\author{
Brian E. Applegate ${ }^{* a}$, Marinko V. Sarunic ${ }^{\mathrm{a}}$, Changhuei Yang ${ }^{\mathrm{b}}$, and Joseph A. Izatt ${ }^{\mathrm{a}}$ \\ ${ }^{a}$ Department of Biomedical Engineering, Duke University, Durham, NC, USA 27708 \\ ${ }^{\mathrm{b}}$ Department of Electrical Engineering, California Institute of Technology, Pasadena, CA, USA \\ 91125
}

\begin{abstract}
Molecular contrast OCT (MCOCT) is an extension of OCT in which specific molecular species are imaged based on their spectroscopic characteristics. In order to improve the sensitivity and specificity of MCOCT, several techniques have recently been introduced which depend upon coherent detection of inelastically scattered light from molecules of interest in a sample. These techniques include harmonic generation, coherent anti-Stokes Raman scattering, and several different forms of pump-probe spectroscopy. We have developed a theoretical framework to facilitate the comparison of different inelastic scattering-based contrast mechanisms for molecular contrast OCT. This framework is based upon the observation that since the noise floor is defined by the reference arm power in a shot-noise limited heterodyne detection system, the relevant comparison among the techniques is isolated to the available molecular-specific signal power. We have derived the value of the molecular contrast signal power for second harmonic generation OCT (SHOCT) and three different pump-probe OCT (PPOCT) techniques. Motivated by this analysis, we have constructed a preliminary ground state recovery pump-probe OCT system, and demonstrated its performance using rhodamine 6G as the MCOCT contrast agent.
\end{abstract}

Keywords: molecular contrast, optical coherence tomography, molecular imaging, nonlinear microscopy

\section{INTRODUCTION}

The genesis of molecular contrast optical coherence tomography (MCOCT) has seen the development of several techniques designed to measure molecular signatures concurrent with OCT imaging. To date linear absorption[1], transient absorption[2], second harmonic generation [3, 4], and coherent anti-Stokes Raman[5] spectroscopies have been demonstrated for MCOCT( see Figure 1 for examples). However, no theoretical or quantitative comparison of the expected signal to noise ratio (SNR) among these different spectroscopies has yet appeared. At this early stage, it is imperative that this comparison be made in order to identify the most promising techniques with the potential to maximize the impact of MCOCT on imaging science. To this end, we have developed a theoretical framework upon which these different techniques may be compared.

The traditional division of spectroscopic techniques into "dark" and "bright" field, based on whether the noise floor is defined by the signal power or some other strong field, is not apropos for the discussion of MCOCT SNR. This is the case because in a shot noise limited OCT system, the noise floor is always defined by the strong local oscillator. For this reason, all MCOCT techniques are bright field. Hence all MCOCT techniques are on equal footing with respect to the noise term in the $S N R$. One minor exception to this rule is the special case where two independent measurements must be performed in order to obtain the MCOCT image, such as in our previous demonstration of phytochrome-

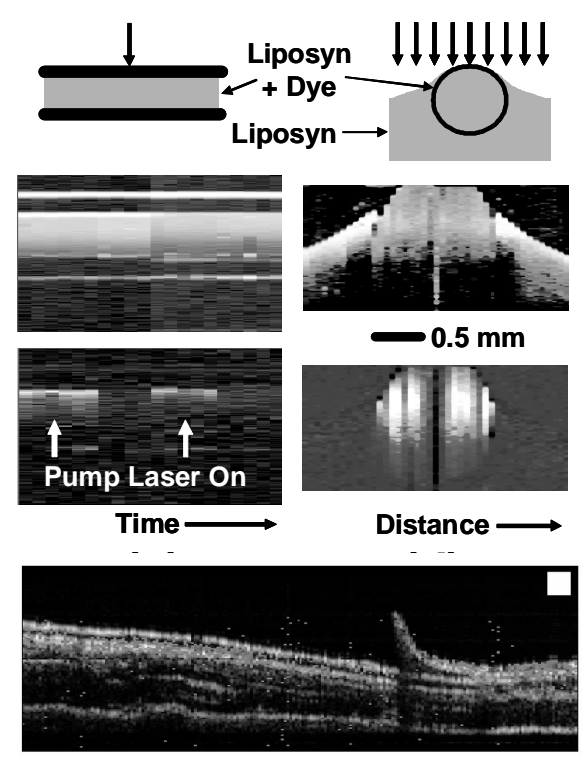

Figure 1 Top) Original PPOCT demonstration in a phantom. [2] Bottom) SHOCT image (green) of fish skin overlayed on top of the OCT image.[3] 
based MCOCT [6]. In this case, the SNR is further reduced by 2 since the noise is given by the statistical sum of the noise from each measurement.

The signal to noise ratio of a Fourier domain OCT system is given by

$$
S N R_{O C T}=\left(\frac{S_{O C T}}{\sigma_{s n}}\right)^{2}=\frac{\rho R_{s} \Delta t}{2 e} P_{s},
$$

where $S_{O C T}$ is the OCT signal, $\sigma_{s n}$ is the standard deviation of the shot noise, $\rho$ is the detector responsivity, $R_{s}$ is the sample reflectivity, $P_{s}$ is the power incident on the sample, $\Delta t$ is the integration time, and $e$ is the electron charge.[7] This equation can fairly simply be rewritten for the molecular contrast power to get

$$
S N R_{M C O C T}=\frac{\rho R_{s} \Delta t}{2 e} P_{M C O C T}
$$

for referenced and non-referenced MCOCT signals, respectively. $P_{M C O C T}$ is the heterodyne signal power of the molecular contrast signal.

\section{RESULTS}

\subsection{Theoretical evaluation of the signal to noise ratio}

As a first example, we derive $P_{\text {MCOCT }}$ for second harmonic OCT (SHOCT) [3-5]. The power in the second harmonic is given by; $P_{2 \omega}=a P_{1 \omega}^{2}$, where $a$ is a function of the spatial profile, temporal profile, and focusing of the laser beam and $P_{1 \omega}$ is the power in the fundamental. The $P_{M C O C T}$ is then simple $P_{M C O C T}=a P_{1 \omega}{ }^{2}$. Since SHOCT has been

\begin{tabular}{|l|l|l|}
\hline Technique & $\boldsymbol{P}_{\text {MCост }}$ & $\begin{array}{c}\text { Expected } \\
\text { SNR }\end{array}$ \\
\hline SHOCT & $a P_{1 \omega}^{2}$ & $54 \mathrm{~dB}$ \\
\hline $\begin{array}{l}\text { PPOCT } \\
\text { scheme 1 }\end{array}$ & $\left(\frac{\sigma_{2} l N_{1}^{0} \sigma_{1} \lambda_{p u} P_{p u}}{2 h c \pi r^{2} f_{0}}\right)^{2} P_{p r}$ & $51 \mathrm{~dB}$ \\
\hline $\begin{array}{l}\text { PPOCT } \\
\text { scheme 2 }\end{array}$ & $\left(\frac{\sigma_{1}^{2} l N_{1}^{0} \lambda_{p u} P_{p u}}{h c \pi r^{2} f_{0}}\right)^{2} P_{p r}$ & $76 \mathrm{~dB}$ \\
\hline $\begin{array}{l}\text { PPOCT } \\
\text { scheme 3 }\end{array}$ & $\left(\frac{\sigma_{3} q_{2,3} l N_{1}^{0} \sigma_{1} \lambda_{p u} P_{p u}}{2 h c \pi r^{2} f_{0}}\right)^{2} P_{p r}$ & $11 \mathrm{~dB}$ \\
\hline
\end{tabular}
demonstrated experimentally using both nonlinear crystals [3-5] as well as in real tissue samples[3, 4], we may compare this result to experimental measurements. Combining equations 1 and 2 along with our expression for $P_{M C O C T}$ we find that the measured $S N R$ of the fundamental should be equal to the second harmonic $S N R$ divided by $a P_{\omega l}$. This was shown to be true experimentally in reference [3], where the authors simultaneously measured the $S N R$ of the fundamental and second harmonic signals. In this case, the measured $S N R_{O C T}=114 \mathrm{~dB}$ and $S N R_{M C O C T} l$ $a P_{\omega l}=113 \mathrm{~dB}$, where the $1 \mathrm{~dB}$ discrepancy was easily explained by the difference in the detector responsivity from $1060 \mathrm{~nm}$ to $530 \mathrm{~nm}$. The quadratic dependence of $S N R_{M C O C T}$ on the power of the fundamental was also demonstrated in references [3-5], thereby verifying the

Table 1 Derived values of the MCOCT power, $P_{\text {MCост }}$. Symbols; $\sigma$ absorption cross-section, $l$ absorption pathlength, $N_{1}{ }^{0}$ ground state population, $\lambda_{p u}$ pump wavelength, $\tau$ pump pulse duration, $f_{o}$ pulse frequncy, $P_{p u}$ pump power, and $r$ focal spot radius. For the SNR we assumed a $200 \mathrm{fs}, 72 \mathrm{MHz}$ laser with $10 \mathrm{~mW}$ of power incident on the sample. We have also assumed a system with responsivity of $0.6 \mathrm{~A} / \mathrm{W}$, time constant $1 \mathrm{~ms}$, a pathlength of $15 \mu \mathrm{m}$, and a focal spot radius of $3.2 \mu \mathrm{m}$. The sample systems consisted of: for SHOCT, pure collagen; for PPOCT, rhodamine 6G with $100 \mu \mathrm{M}$ concentration. fundamental equation from which the MCOCT power was derived .

As a second example, pump-probe OCT (PPOCT) encompasses a class of techniques in which the OCT light serves as a probe beam tuned to a molecular resonance of interest whose population is modulated by a separate pump beam (Fig. 2). As a coherent analog of fluorescence, PPOCT has the potential to be utilized in much the same way that fluorescence microscopy is utilized today. Since PPOCT exploits the same fundamental molecular property, linear absorption, as fluorescence microscopy, functional extensions such as FRAP and FRET are also amenable to PPOCT. In addition, one may also tap into the vast experience developed over decades in molecular spectroscopy to gather molecular dynamics information from pump-probe experiments; $e$. g. excited state lifetimes, quantum yields, and vibrational energies.

For pump-probe OCT, the derivation of $P_{M C O C T}$ requires explicitly tracking the population changes in the states utilized in the pump-probe scheme. Here, we consider the three most prominent two-photon pump-probe schemes depicted in 
Figure 2. Higher order pump-probe schemes require multiple spontaneous processes, which will invariably lead to significant losses in the efficiency. Scheme 1 is the standard pump probe experiment, where the pump excites molecules from state 1 into state 2 and the probe interrogates the population of state 2 by monitoring the transient absorption from state 2 to state 4 . Scheme 2 is a ground state recovery pump-probe approach in which the pump excites molecules from state 1 to state 2 , and the probe interrogates the population of state 1 by monitoring the transient bleaching of the 1-2 transition. Scheme 3 is similar to scheme 1, except that a curve crossing in the excited state transfers population from state 2 to state 3 and the population of state 3 is monitored with the 3-5 transition. The efficiency of the curve crossing is given by the quantum efficiency, $q_{2,3}$. Scheme 3 is the technique utilized in the first demonstration of PPOCT in methylene blue. In methylene blue the transition from state 2 to state 3 was a spin forbidden singlet-triplet transition.

Table 1 lists the expressions for $P_{M C O C T}$ we have derived for the three pump-probe schemes noted above, as well as for SHOCT. Since $P_{M C O C T}$ differs for these three schemes only by the physical constants $\sigma_{1}, \sigma_{2}, \sigma_{3}$, and $q_{2,3}$, in order to determine which scheme has the best $S N R$, we must estimate the values of these constants. The quantum efficiency, $q_{2,3}$, is always less than one, and is typically in the range of $10^{-3}-10^{-5}$. For ICG[8] and rhodamine 6G[9] the singlet-triplet quantum efficiencies are $2 \times 10^{-6}$ and $8 \times 10^{-3}$, respectively. Hence scheme 3 will in general have $3-5$ orders of magnitude worse SNR compared to schemes 1 and 2. Typically, excited state absorption cross-sections are smaller than ground state absorption cross-sections. For instance in rhodamine $6 \mathrm{G} \sigma_{1}=1.50 \times 10^{-16} \mathrm{~cm}^{2}$ and $\sigma_{2}=6.9 \times 10^{-17} \mathrm{~cm}^{2} .[10]$ Hence, from an SNR standpoint, scheme 2 is slightly better than scheme 1. From a practical standpoint, scheme 2 only requires a single laser which obviates the need to synchronize two laser systems. Additionally, scheme 2 only requires knowledge of the absorption cross-section and wavelength of the 1-2 transition, which are typically available for standard molecular contrast agents.

Column 3 in Table 1 gives expected SNR for SHOCT and the three PPOCT techniques discussed here, under the conditions noted in the caption. For SHOCT we consider a pure collagen sample as characterized in reference [11]. Note that the $S N R$ value for pure collagen should be considered an upper value for several reasons. No biological sample is pure collagen. We have not explicitly considered the losses due to destructive interference between second harmonic light generated at different points in the tissue. We have implicitly assumed that the laser polarization is aligned with the molecular hyperpolarizability, thereby providing the maximum second harmonic response. For the PPOCT techniques we consider rhodamine 6G via each scheme. Clearly from table 1 gsrPPOCT is has the best predicted SNR of all of the techniques considered, by a significant margin. As predicted with the order of magnitude arguments above, the SNR for the 3 PPOCT schemes goes as scheme $2>$ scheme 1> scheme 3. The development of gsrPPOCT has the potential to allow MCOCT imaging with a number of common molecular contrast agents, including the transfectable proteins like DsRed. There is also the potential to utilize infrared absorbing contrast agents which cannot be used for fluorescence microscopy due to their poor fluorescence quantum yields.

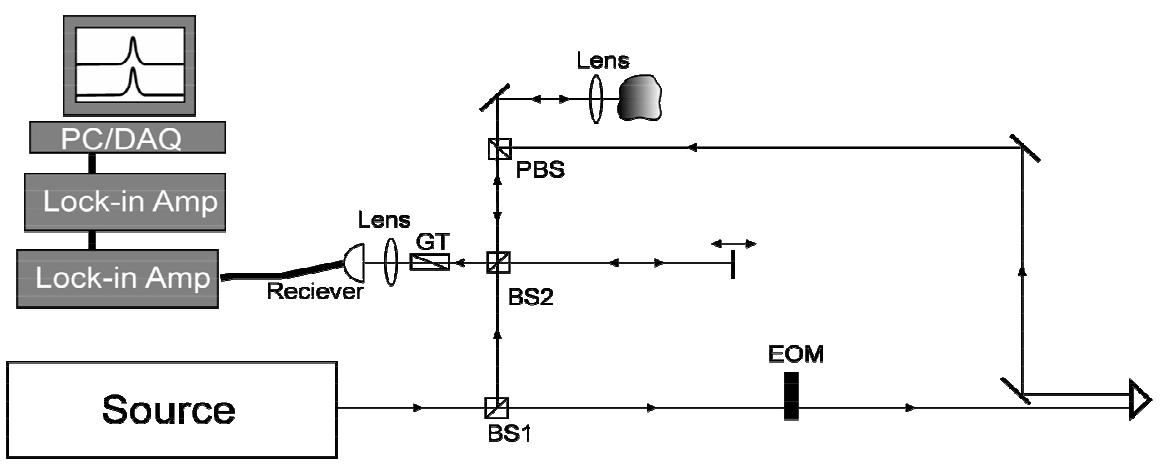

Figure 3. Schematic diagram of the ground state recovery PPOCT setup. Abbreviations: Glan-Thomson polarizer (GT), beam splitter (BS), electro-optic modulator (EOM), polarizing beam splitter (PBS).

\subsection{Optical setup and testing of ground state recovery PPOCT}

Based on this analysis, we have constructed a ground state recovery PPOCT system illustrated in Fig. 3. For an initial demonstration, we have chosen rhodamine $6 \mathrm{G}$ as the contrast agent, using a frequency-doubled $100 \mathrm{fs}$ $\mathrm{Nd}$ :Glass laser pulse for both the pump and probe fields. Although the pump-probe wavelength of $530 \mathrm{~nm}$ is unfavorable for OCT imaging, it does facilitate a direct comparison with fluorescence microscopy. Figure 3 shows a 
schematic diagram of the experimental setup. The system is a standard time-domain free space OCT setup, with additional elements to accommodate the pump beam. The pump beam is split off at BS1. The pump is amplitude modulated at $20 \mathrm{kHz}$ with an electro-optic modulator, and its polarization is rotated by $\pi / 2$. The pump is then delayed by an entire cycle of the laser (to ensure incoherence with the probe beam) and recombined via a polarizing beam splitter with the sample arm of the interferometer. The delay between the pump and probe is maintained at several hundred picoseconds. The MCOCT signal is detected by lock-in demodulation of the signal at the difference frequency of the Doppler shift and pump modulation frequency. The $S N R_{O C T}$ of the system was measured to be $87 \mathrm{~dB}, 6 \mathrm{~dB}$ less than the shot noise limit for the sample arm power of $120 \mu \mathrm{W}$ and acquisition bandwidth of $33 \mathrm{kHz}$. Using a sample consisting of a $58 \mu \mathrm{M}$ aqueous solution of

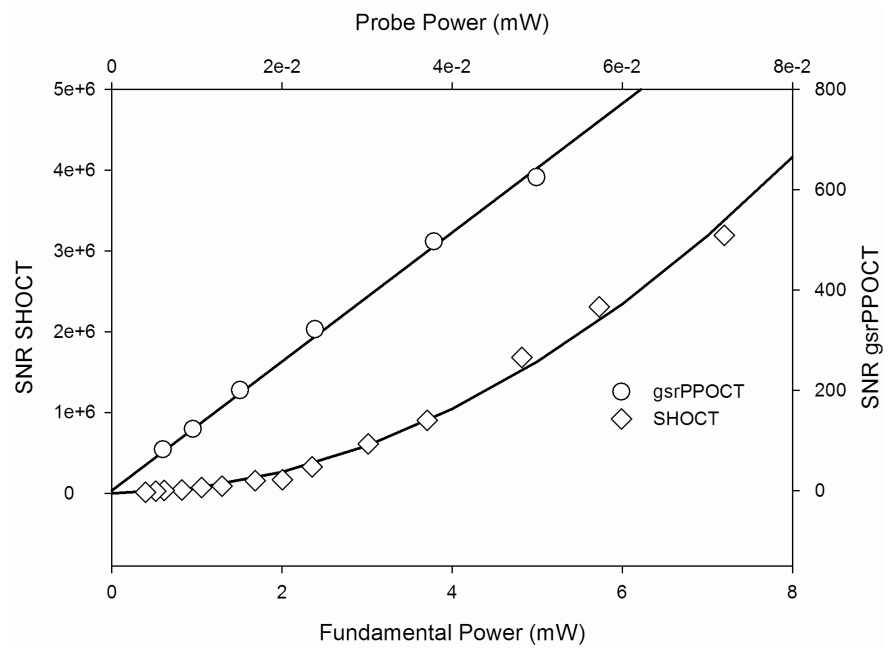

Figure 4 Plot of SNR vs. sample arm power for gsrPPOCT and SHOCT. rhodamine $6 \mathrm{G}$, sandwiched with a pathlength of $780 \mu \mathrm{m}$, between a microscope cover slip and a mirror, we measured an $S N R_{M C O C T}$ of $56 \mathrm{~dB}$, with a pump power of 3.35 $\mathrm{mW}$. The $S N R_{M C O C T}$ predicted for these experimental conditions based on the contents of Table 1 was $61 \mathrm{~dB}$.

Figure 4 is a plot of probe power (sample arm power) vs. SNR for gsrPPOCT obtained using the rhodamine setup. For reference, the same plot is provided for SHOCT. As can be seen from the plot and as the theory predicts, the SNR of gsrPPOCT varies linearly with the probe power. In comparison, the SNR for SHOCT varies as the square of the fundamental power (sample arm power).

\section{CONCLUSIONS}

In conclusion, we have developed a theoretical framework upon which to compare different molecular contrast techniques for MCOCT. Using this framework, we have derived the expected SNR for three different pump-probe schemes and found the ground state recovery PPOCT scheme to have superior SNR to the other two schemes considered. Based on this analysis, we have constructed a ground state recovery PPOCT system and begun the process of testing and refining the optical setup.

\section{ACKNOWLEGEMENTS}

We gratefully acknowledge support for this work through a grant from the National Institute of Health, R01 EB000243. BEA acknowledges support via a Kirschstein postdoctoral fellowship, F32 EB004237.

\section{REFERENCES}

1. Morgner, U., et al., Spectroscopic optical coherence tomography. Opt. Lett., 1999. 25(2): p. 111-113.

2. Rao, K.D., et al., Molecular contrast in optical coherence tomography by use of a pump-probe technique. Optics Letters, 2003. 28(5): p. 340-342.

3. Applegate, B.E., et al., Polarization resolved second harmonic generation optical coherence tomography in collagen. Opt. Lett., 2004. 29(19): p. 2252-2254.

4. Jiang, Y., et al., Second-harmonic optical coherence tomography. Opt. Lett., 2004. 29(10): p. 1090-1092.

5. Vinegoni, C., et al., Nonlinear optical coherence enhancement for optical coherence tomography. Opt. Exp., 2004. 12(2): p. 331-341. 
6. Yang, C., et al., Protein-based molecular contrast optical coherence tomography with phytochrome as the contrast agent. Opt. Lett., 2004. 29(12): p. 1396-1398.

7. $\quad$ Choma, M.A., et al., Sensitivity advantage of swept-source and Fourier-domain optical coherence tomography. Optics Express, 2003. 11(18): p. 2183-2189.

8. Gratz, H., et al., Photo-Isomerisation, Triplet Formation, and Photo-Degradation Dynamics of Indocyanine Green Solutions. J. Photochem. Photobio. A, 1999. 128: p. 101-109.

9. Riendl, S. and A. Penzkofer, Triplet quantum yield determination by picosecond laser double-pulse fluorescence excitation. Chem. Phys., 1996. 213: p. 429-438.

10. Delysse, S., J.-M. Nunzi, and C. Scala-Valero, Picosecond optical Kerr ellipsometry determination of $S_{l}-S_{n}$ absorption spetra of xanthene dyes. Appl. Phys. B, 1998. 66: p. 439-444.

11. Stoller, P., et al., Quantitative Second-Harmonic Generation Microscopy in Collagen. Applied Optics, 2003. 42: p. 5209.

*brian.applegate@duke.edu ; phone 1919 660-5588 